\title{
LA RESOLUCIÓN DE PROBLEMAS PUEDE SER SOMETIDA A DEBATE: FANTASMAGRAMAS PERFORMATIVOS DE CRISIS EN LA REFORMA EDUCATIVA STEM $^{2}$ DE ESTADOS UNIDOS
}

\section{Can problem-solving be problematized: performative phantasmagrams of crisis in US STEM education reform}

Lei ZHENG

Universidad de Wisconsin-Madison

Recibido: 3 de febrero de 202I. Envío a informantes: 8 de febrero de 202I.

Aceptación definitiva: 9 de mayo de 2021

RESUMEN: Aceptando que los estudios del currículum suelen centrarse en la organización, planificación y ordenamiento de los conocimientos, ya sea de manera normativa o crítica, la historia del currículum consiste menos en examinar el currículum del pasado que en estudiar las prácticas históricas que se entrecruzan en las formas de organizar el conocimiento. Con el giro producido hacia el enfoque performativo en los estudios científicos, este artículo debate sobre las formas de imaginar y materializar «situaciones»y «problemas» expresados en la educación STEM como crisis de la futura supervivencia por medio de una historiografía performativa. El artículo comenzará presentando los estudios de la ciencia poshumanista sobre la performatividad y los conceptos de «fantasmagrama» de Michelle Murphy (2017), para responder de las condiciones conceptuales-materiales-afectivas de los instrumentos que configuran el espacio tiempo y dan estabilidad a los fenómenos. Así, primero abordará los estudios existentes en historia y sociología de la ciencia para debatir cómo se inventó «la situación de la vida real» por medio de experimen-

NT.- Fantasmagramas bien podría traducirse como técnicas de representación visual.

NT.- STEM es el acrónimo de Science, Technology, Engeenering and Mathematics, utilizado por la comunidad científica de procedencia anglosajona para incluir estos cuatro campos disciplinares académicos (Ciencia, Tecnología, Ingeniería y Matemáticas), que a su vez agrupan y acogen entre sí a otras disciplinas más específicas. 
tos militares y científicos estadounidenses que la sustituyeron como condición de su producción durante el periodo de la guerra mundial y la posguerra. A continuación, propondré dos experimentos (el experimento de conducta de pánico de Mintz y el Movimiento Espacial de la Tierra) para examinar cómo el fantasma de la crisis fue coconfigurado con la simulación científica de resolución de problemas colectiva a ambas escalas micro y macro durante los años 1950-1970. Por último, se presentará cómo este fantasmagrama de la crisis empujó el currículum de anticipación y la pedagogía de la «resolución de problemas» para preparar a los estudiantes estadounidenses para la supervivencia global en y después de los años i980.

PALABRAS ClAVE: resolución de problemas; performatividad poshumanista; historia del currículum; reforma USA de la educación STEM; fantasmagrama de la crisis.

ABSTRACT: If we can agree that curriculum studies generally focus on organizing, planning, and arranging knowledge, either in a normative or a critical way, then curriculum history is less about looking at the past curriculum than the historical practices that are entangled in the ways that knowledge is organized. With the turn to performative approach aroused in science studies, this article problematizes the ways «situations» and «problems» embodied in STEM education are imagined and materialized as crisis of future survival through a performative historiography. The article will begin with introducing post-humanist science studies of performativity and Michelle Murphy (2017)'s concepts «phantasmagram» to accounting for the conceptual-material-affective conditions of apparatuses that configure spacetime and stabilize phenomena. Then, it will first draw upon existing scholarship in the history and sociology of science to discuss how the "real-life situation» was invented through US military and scientific experiments that substituted it for the condition of its production during the war and post-war era. Following that, I will explore two experiments (Mintz' experiment of panic behavior and Spaceship Earth Movement) to examine how the phantasma of crisis was coconstituted with the scientific simulation of collective problem-solving at both micro and macro scales during the 1950s-1970s. Lastly, it will introduce how this phantasmagram of crisis animated the anticipatory curriculum and the pedagogy of problem-solving to prepare American students for global survival in and after the ig8os.

KEY WORDS: problem-solving; post-humanist performativity; curriculum history; US STEM education reform; phantasmagrams of crisis.

\section{La problemática de la resolución de problemas en la reforma educativa de los STEM de Estados Unidos}

En Diseños y Modelos, la mayor parte de la atención se presta a cómo discriminar entre ciencia y tecnología... Esta estrecha unión también representa al método científico como un proceso de resolución de problemas en contraste con una búsqueda racional del conocimiento que defiende o rechaza ideas que tantean 

LEI ZHENG

sobre la naturaleza. Sugiere que se inician los esfuerzos científicos como respuesta a problemas que deben resolverse (cursiva mía) sin prestar atención a la idea que con frecuencia es la búsqueda del conocimiento por sí mismo. (Engineering in $K$-12 Education, 2009, p. 262)

$\mathrm{Al}$ aprender a resolver problemas en matemáticas, los estudiantes deben adquirir formas de pensar, hábitos de constancia y curiosidad, y confianza en situaciones imprevistas (cursiva mía) que les serán muy útiles fuera de la clase de matemáticas. En la vida diaria y en el lugar de trabajo ser capaz de resolver bien problemas puede representar grandes ventajas. (Principles and Standards for School Mathematics, 2000, p. 52)

$\mathrm{D}$ ENTRO DEL DISCURSO DE LA REFORMA ESCOLAR de los STEM en Estados Unidos, la resolución de problemas está especialmente reconocida como el corazón de la reforma educativa del siglo xxi. Se dice que no solo alimenta la curiosidad de los estudiantes, sino que para el futuro también garantiza la seguridad global de cada individuo y del Estado-nación (NCTM, 200o; US Department of Education, 20I6). Uno de los rasgos distintivos de la educación STEM es la integración de la ingeniería y la tecnología en el currículum de ciencia y matemáticas, que define la ingeniería como proceso de resolución de problemas y las tecnologías como el producto de la resolución de problemas (NGSS Lead States, 2013; NAE y NRC, 2009). El aula de STEM está diseñada para facilitar un entorno de aprendizaje que simula los retos del «mundo real» para desarrollar en los estudiantes la más elevada disposición cognitiva para adaptarse y sobrevivir a un mundo desconocido (US Department of Education, 20I6; ver también stemecosystem.org, https://ambitiousscienceteaching.org).

La resolución de problemas ha formado parte, aunque no central, de la educación de los Estados Unidos desde el auge de las ciencias de la educación a comienzos del siglo xx. Los investigadores en educación (e. g. Popkewitz, 2004; Noble, 1989) han criticado que la aceptación de una naturaleza dada y la estructura del razonamiento matemático y científico incorporado a la resolución de problemas provoca que los estudiantes estén sometidos a la autoridad de la «pericia» científica y les hace tener disciplina y control de sus propias mentes en el lenguaje de los empresarios, como «ser responsable de su propio aprendizaje» o «domina tus propios recursos cognitivos». La flexibilidad del aprendizaje que con frecuencia se ha subrayado en la resolución de problemas también ha sido criticada, ya que se pide a los estudiantes que tengan control sobre sí mismos para ser más adaptables al orden establecido, lo que sería muy deseado por los empresarios. Noble (I989) también señala que el auge de la resolución de problemas en el sistema educativo de los Estados Unidos en los años i980 procede de la investigación cognitiva militar, que trata de convertir a los humanos en componentes adecuados para un rendimiento óptimo del sistema armamentístico y, por ello, el objetivo de «educar» a alguien que resuelve problemas no busca aplaudir la capacidad de los seres humanos, sino más bien que puedan ser prescindibles. 
Sin embargo, el cuestionamiento que existe sobre la resolución de problemas en la investigación educativa principalmente se centra en la segunda parte de esta frase, es decir, en cómo resolver, y se ha prestado menos atención a la primera parte, es decir, qué situaciones y problemas son de interés actual y cómo han llegado a importar. En este artículo se plantea que la literatura científica sobre la reforma educativa estadounidense de los STEM sitúa la resolución de problemas en un entorno de crisis generalizada en la que se supone que los estudiantes han de afrontar conflictos, riesgos y peligros en cualquier momento. Para unir seguridad e innovación, esta literatura científica ha abordado la pedagogía de la resolución de problemas cognitiva y afectivamente al menos de dos formas. En primer lugar, la resolución de problemas prepara para el mundo real ofreciendo un repertorio de situaciones peligrosas y prescribiendo el proceso de cómo superar el peligro. Por ejemplo, los cuentos de hadas narrados en la literatura infantil, como Los Tres Cerditos y Caperucita Roja, son adaptados como lecturas STEM (e. g., Troupe, 2019) para motivar el interés de los niños hacia los STEM, como una herramienta crítica para proteger el yo infantil vulnerable del peligroso lobo/enemigo en su vida diaria. En segundo lugar, la resolución de problemas es adelantarse a las crisis imaginando las situaciones y problemas desconocidos de forma proactiva. Los programas de educación STEM se crean dentro del marco del modelo DARPA (Defense Advanced Research Projects Agency-Agencia de Proyectos de Investigación Avanzada de Defensa del Pentágono de Estados Unidos), modelo que dirige la investigación para resolver problemas específicos e inmediatos «basados en disponer de una visión clara del éxito, incluso cuando todavía no se conocen todas las piezas del rompecabezas» (Shilling, 20I4). Por ejemplo, varios campos espaciales y proyectos patrocinados por la NASA proporcionan simulación de aventura de temática militar en la que se supone que los estudiantes experimentan diversión y peligro mientras sobreviven y llevan a cabo una misión en el espacio exterior. A través de una «oportunidad de peligro», se pide a los estudiantes que cambien su vergüenza y miedo al fracaso por arrestos para asumir riesgos y cosechar las mayores recompensas» (US Department of Education, 2016; NAS, NAE e IM, 2007).

Este artículo en especial cuestiona las formas en que las «situaciones» $\mathrm{y}$ "problemas» incorporados a la educación STEM se imaginan y concretan como crisis de futura supervivencia para elaborar una forma concreta de resolver problemas. Lo hace explicando históricamente cómo se inventó la «situación del mundo real» en los experimentos científicos de los Estados Unidos y más tarde se transfirió a la investigación social y educativa para hacer de la resolución de problemas el estándar troncal del currículum del milenio en los Estados Unidos.

El artículo comenzará exponiendo los estudios de la ciencia poshumanista sobre la performatividad y los conceptos «fantasmagrama» de Michelle Murphy (2017) en la historia del currículum para explicar las condiciones conceptuales-materiales-afectivas de los aparatos del conocimiento. A continuación, aprovechará los estudios existentes en la historia y sociología de la ciencia para debatir cómo se inventó la «situación de la vida real» mediante experimentos 
militares y científicos que la sustituyeron por sus condiciones durante la guerra y la posguerra. A continuación, examinaré dos experimentos (el experimento de Mintz sobre la conducta de pánico y el Movimiento Espacial de la Tierra) para estudiar cómo se co-constituyó un fantasma de crisis con la simulación científica de la resolución de problemas colectiva tanto a escala micro como macro entre los años 1950 y 1970. Por último, se presentará cómo este fantasmagrama de crisis impulsó el currículum anticipatorio y la pedagogía de la resolución de problemas para preparar a los estudiantes de los Estados Unidos para la supervivencia global en y después de los años 80 .

\section{Performatividad, fantasmagrama e historia del currículum}

Si aceptamos que los estudios del currículum suelen centrarse en la organización de los conocimientos, ya sea de forma normativa o crítica, entonces la historia del currículum, como se ejemplifica en este número especial de la revista Historia de la Educación, no se fija tanto en el currículum del pasado como en las prácticas y efectos históricos que se entrecruzan en las formas como se organiza el conocimiento. Para responder a esta pregunta sobre las condiciones y políticas del conocimiento y la subjetividad, la teoría de la performatividad, que se ha desarrollado más en los estudios de la ciencia poshumanista, aporta una profunda intervención de estas nociones fundamentales en la epistemología de Occidente que permanecen inamovibles en la construcción del currículum, como la representación (frente a la realidad), la agencia, la causalidad y la responsabilidad.

Mientras las ciencias sociales continúan interactuando con los problemas del mundo real «viéndolos» (preferentemente en tiempo real) y, de este modo, resolviéndolos 3 , Karem Barad (2003, 2007) interrumpe esta persistencia del presentismo y el representacionismo al desplazar tácticamente «interacción» con «intra-acción». Como ella sostiene,

Los fenómenos no señalan simplemente la inseparabilidad epistemológica de «observador» y «observado»; al contrario, los fenómenos son la inseparabilidad ontológica de «componentes» agencialmente intra-actuantes.... Una intra-acción específica (que implica una configuración material específica del «aparato de observación») produce un corte agencial (en contraste con el corte cartesiano -una distinción inherente- entre sujeto y objeto) provocando una separación entre «sujeto» $\mathrm{y}$ «objeto» (Karen Barad, 2003) 4 .

Ver otra crítica histórica de este número en Bernadette Baker (2017).

4 NT. - El realismo agencial es una teoría propuesta por Karen Barad, la cual proviene de la intersección entre estudios de género y la Teoría del Actor-Red. En el ámbito de la filosofía y la sociología, se denomina agencia a la capacidad que posee un agente (una persona u otra identidad) para actuar en un ámbito del mundo. 
El enfoque performativo posthumanista propone una separación a priori de «cómo conocemos» y «qué sabemos» reorientando nuestra atención a los «cortes agenciales» que hacen posible esta separación específica y la co-constitución de ambos, el cognoscente y el conocido (Schrader, 20Io). Nos invita a repensar la cuestión histórica de cómo llegaron a producirse «los problemas reales del mundo». Esta pregunta ha sido retirada del conocimiento escolar que solo prioriza las soluciones o separa la cuestión epistemológica de las soluciones del estatus ontológico de los problemas. Lo que importa como problema no es una mera cuestión conceptual/teórica, sino que, inspirándose Barad (2007) en la lectura difractiva de Bohr, «se define por su inserción como parte del plan material -que incluye la instrumentación (por ejemplo, placas fotográficas, punteros o dispositivos de lectura digital) que asigna valores concretos a las propiedades específicamente definidas y que pueden ser leídos por un observador humano como cargados de significado-» (p. I43). Más importante aún, la disposición material del discurso es también un fenómeno que se constituye, estabiliza y reconfigura históricamente. Conocer un problema es recomponer el mundo dentro del mundo.

La configuración discursiva-material de los «problemas del mundo real» también puede considerarse como una especie de fantasmagrama, término tomado de Michelle Murphy (2017) para denominar la inversión afectiva y los consiguientes imaginarios de las prácticas cuantitativas de las ciencias sociales (pp. 24-25). En su libro sobre la economización de la vida, ella analiza cómo el modelo de transición demográfica y las estimaciones de la natalidad evitada como fantasmagramas de la población estaban formados y eran producto de los imaginarios y sentimientos que daban vida a los números y orientaban la facticidad mientras que, al mismo tiempo, procuraban borrar sus propias condiciones de creación. Los fantasmagramas son performativos, ya que no solo hacen aparecer una proyección del mundo mediante aparatos discursivos-materiales que se han inventado de forma muy precisa, sino que su proyección también establece un método específico de cómo pensar y conocer. Si bien el fantasma y las mutaciones iterativas de los aparatos que son productivos y parte de él no están completamente presentes ni totalmente ausentes, son, como arguye Schrader (20I0), «agencialmente reales»; contribuyen a su propia materialización y nos piden rendir cuentas. La responsabilidad en las prácticas científicas depende de cómo sus 'agencias' son tenidas en cuenta.

Inspirado en los trabajos ya existentes sobre la ilusionista evaporación científica del mundo y sus irracionales temáticas en el periodo de la Guerra Fría (véase Murphy, 2017; Jachie Orr, 2006; Erickson et al., 2013), este artículo analizará cómo después de la Segunda Guerra Mundial se construyeron determinados aparatos de comunicación (como simulación, modelización, diagrama y otros experimentos físicos y mentales) para conjurar los fantasmagramas de la crisis que generan imaginarios colectivos y ponen en marcha llamativas actuaciones metafóricas para reorganizar el mundo y reorientar acciones y modos de pensar. Lo que nos importa en esta investigación es menos si debemos o no «imaginar» o servirnos de enfoques estéticos en el trabajo intelectual que cómo evaluar los efectos de los 
imaginarios que se han elaborado y consolidado como reales mediante el uso de ciertos aparatos de conocimiento.

\section{Invención científica y militar de «situaciones» para la resolución de problemas}

La identificación de situaciones a menudo se acepta como el primer paso para la resolución de problemas, como si las situaciones estuvieran ahí de forma natural esperando a ser descubiertas por quienes resuelven los problemas. Reflexionando a partir de la teoría de la performatividad poshumanista, este artículo toma las situaciones como una configuración discursivo-material del espacio tiempo para que los problemas particulares sean visibles e inteligibles. Los aparatos científicos que incorporan y actualizan las situaciones históricamente han sido inventados con jerarquías políticas y epistémicas específicas.

Las situaciones como manifiestos metodológicos se han inventado y materializado en la investigación de los Estados Unidos a través del actual colonialismo de colonos'. En el libro How Reason Almost Lost its Mind, Erickson et al. (2013) (Cómo la razón casi perdió la cabeza) toman los atolones micronesios como un ejemplo para mostrar cómo un lugar real con toda su población se convirtió en un laboratorio en parte irreal para los experimentos nucleares y sociopsicológicos de los Estados Unidos inmediatamente después de la II Guerra Mundial. Micronesia se convierte en una situación ideal en la que la vida real de los locales quedó eliminada y sustituida por los residuos de las pruebas nucleares y la información social y psicológica (p. Io). Otros lugares parecidos -islas, campos de cultivo, aldeas- se convirtieron en lugares fantasma pues eran tanto artificiales como reales, y los efectos que produjeron estos experimentos artificiales en esos lugares y en esas personas no fueron tenidos en cuenta como condiciones de estos experimentos. Se convirtieron en meros códigos y datos para validar los informes científicos que se elaboraron con la observación tecnológica y la intervención de los lugareños sin su reconocimiento. Los datos recopilados mediante infraestructuras coloniales de los colonos también fueron tratados en los laboratorios para producir una ley universal para toda la vida. Como Murphy (20I7) destaca, «el negocio de la conquista» en la Argelia colonizada se naturalizó al aportar registros coloniales como pruebas científicas del crecimiento de la población en la curva $-S$, que se podía repetir con moscas de la fruta en botellas bajo control (Fig. I). En el periodo de la Guerra Fría poscolonial, «los países en desarrollo» se convierten, de nuevo, en situaciones experimentales de la población y otras formas de intervención social para validar las teorías científicas sociales de Estados Unidos (véase también en Bonneuil, 2000; Tsing, 2005).

Michelle Murphy lo define en su conferencia como «no un evento sino una estructura que se extiende a través de las infraestructuras y el tiempo». 
Una situación en el experimento científico social es un concepto epistemológico particular. Se define como «un lugar de incertidumbre -un problema- que podría resolverse en armonía utilizando la comprensión y el experimento adecuados» (Dewey, citado en Erickson et al., 2013, p. II3). Se basa en la incertidumbre y la confusión como factores desencadenantes de un cambio caracterizado por el equilibrio y la coherencia -un orden racional-. Se inventa una situación como un sistema compuesto en el que las conductas irracionales del hombre podrían ser controladas por un método racional, lo que significa «un análisis ordenado, una metodología bien pensada, la incorporación del error de comportamiento respecto al funcionamiento sistemático, la codificación minuciosa de los componentes, y el alisamiento de lo inesperado (acto único) en favor de lo predecible (modelo general)» (p. II9).

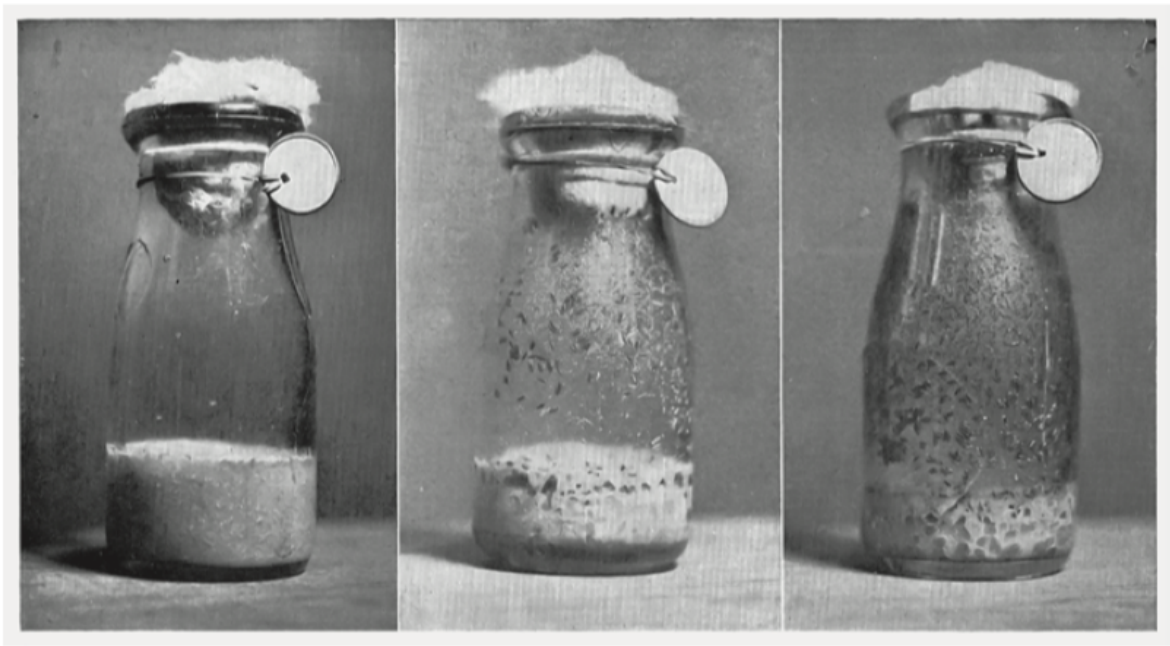

Fig. I. La botella de Drosophila de Raymond Pearl en tres momentos. (Murphy, 20I7; la fuente original: R. Pearl, Biología del crecimiento de la población, 1930).

Como otros conceptos teóricos utilizados en los experimentos científicos, las situaciones adoptan forma material. Erickson y otros (2013) ofrecen un interesante análisis de la invención de Robert Free Bales de la «sala especial» para observar cómo se resuelven problemas de grupo de «situaciones de la vida real». Este tipo de sala especial conocida como «microaula» todavía existe en escuelas y universidades actuales y suele considerarse como la más avanzada tecnológicamente. Esta sala especial (Fig. II) se construyó de tal manera que podía eliminar todo tipo de anomalías, ruidos o errores que se consideraban evitables en la codificación de los comportamientos humanos a fin de producir una «situación normal» de interacción social en la que los procesos irracionales del trabajo en grupo pudieran manifestarse «libremente», ser atendidos y debatidos. Por tanto, la situación estaba altamente controlada para ser «libre», y por ello «natural»y «normal», lo 

LEI ZHENG

que se define por «toda la gama de tendencias internas o posibilidades del proceso de interacción» (Bales, I95I). Al mismo tiempo, se suponía que el control debía ser realizado por observadores «tecleando sin parar, sin pensar en nada, actuando como instrumentos pasivos» para demostrar un proceso de resolución de problemas -o «movimiento de fase» que se producía solamente bajo determinadas condiciones (Bales, I95I)-. Los aparatos experimentales -tanto la sala física como su diseño- producían un corte agencial que separaba, pero al mismo tiempo también daba forma tanto al observador como a la situación observada.

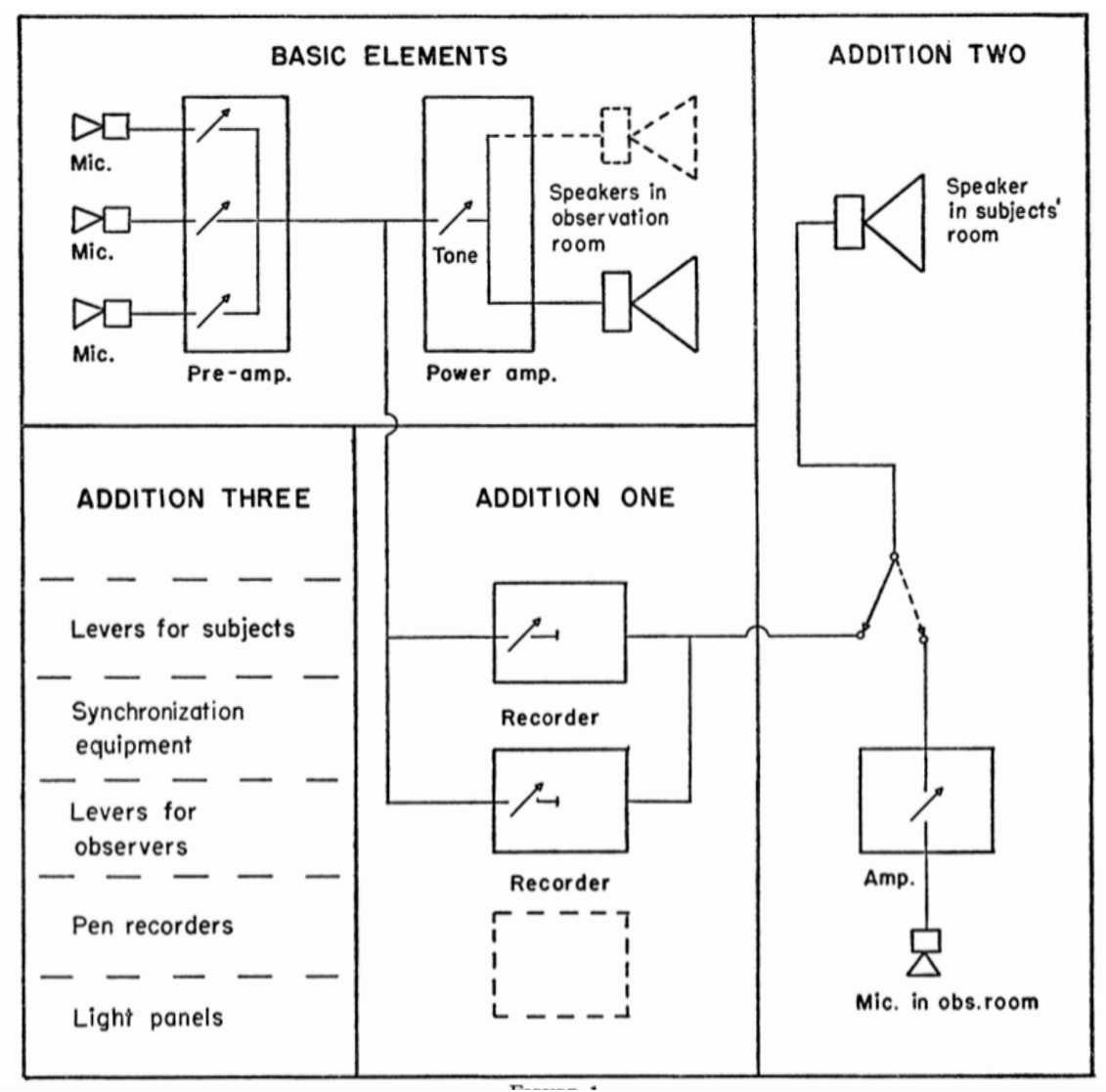

Fig. II. El diseño de Bales de una sala de observación (Bales, I954).

Para plantear una situación «normal» para observar una resolución de problemas no solo se controló muy bien a los observadores y a la sala, sino que también se seleccionó de manera diferenciada a los grupos observados. Por ejemplo, los grupos no debían acoger «personas de inteligencia subnormal o personalidades gravemente perturbadas». Los participantes han de ser adultos, o casi adultos, miembros de nuestra propia cultura occidental... «que hablen inglés, posean 
educación escolar, etc.». Y cada grupo debe recibir un mínimo de presión para mantener su solidaridad (Bales, I95I). Por tanto, el «resultado» de esta situación atendiendo a lo que era una resolución de problemas ya había sido prescrito en la elaboración diferencial de la situación.

Estas condiciones desaparecieron cuando se transfirió la codificación de la interacción de los grupos pequeños en una situación social minúscula y se aplicó a todas las interacciones sociales del mundo real, aunque esta aplicación universal de los hallazgos experimentales podría no estar respaldada por sus inventores. Como sostiene Orr (2006)

Las abstracciones conceptuales que sostienen esta miniaturización no son, inicialmente, abstracciones matemáticas, aunque organizan y más tarde permiten el análisis cuantitativo y estadístico. Inicialmente las abstracciones se fundan en otro lugar, en las operaciones simbólicas bastante comunes de la metáfora y la analogía. (p. 92)

La metáfora y la analogía funcionan como un fantasmagrama en los experimentos de las ciencias sociales. Su funcionamiento no solo es simbólico sino también material. Como fantasmagramas, permiten transformar los actores y fenómenos sociales en imaginarios culturales que pueden ser enunciados en el lenguaje de las ciencias sociales y materializados en los laboratorios como «datos brutos» para la futura elaboración experimental y teórica.

\section{Del experimento de Mintz al Movimiento Espacial de la Tierra. ¿Cómo sobrevive una multitud a un espacio y tiempo finitos?}

Para estudiar más profundamente cómo la resolución de problemas y sus solucionadores llegaron a importar como efectos específicos de los fantasmagramas de los problemas del mundo real, esta sección se centra en dos acontecimientos en apariencia dispares relacionados con la misma abstracción conceptual del problema del mundo. Se marca cómo se produjo un fantasmagrama concreto -el problema de la supervivencia de una multitud en un espacio y tiempo finitos (por ejemplo, el imaginario de los atascos de tráfico en hora punta)- y cómo se desplazó a través de las fronteras de las disciplinas y las espacio-temporales mientras que, al mismo tiempo, esos límites se fueron construyendo de manera performativa.

\section{I. La botella experimental de Mintz de comportamiento no adaptativo}

Analizando la elaboración discursiva-material del pánico en la psicología social de los años 1950, Orr (2006) observa que «el 'grupo' parece ser una entidad simbólica y psicológica cada vez más flexible» y la «multitud» es definida como 
«una forma concreta de organización para la solución colectiva de problemas» (Swanson, 1953, citado en Orr, 2006, p. 13). Uno de los experimentos clásicos sobre la conducta de la multitud fue dirigido por Alexander Mintz en $1950^{6}$. Después de su experimento Mintz planteó recopilar «datos de la vida real» para lograr una verificación completa de esta teoría.

El experimento de Mintz buscaba simular cómo los individuos podrían sobrevivir a una situación de pánico, como incendios en teatros o atascos de tráfico, con multitudes con muy pocas salidas. Calificó la conducta de la gente «que bloqueaba las salidas empujando a que los individuos se quemaran o fueran pisoteados» como no adaptativa (I95I). El comportamiento no adaptativo significaba que los individuos no eran capaces de adaptarse a su entorno y, siguiendo la ley biológica de la selección natural, la no adaptación causaría desorden y extinción.

Mintz rechazó la explicación por entonces dominante de que la conducta no adaptativa de la multitud en situación inducida de pánico se debía a la excitación emocional o a aquellos que vinculaban esta idea a la ideología del fascismo. Utilizó soldados y atletas como ejemplos para mostrar que los individuos podrían comportarse de forma adaptativa en grupo manteniendo al mismo tiempo las emociones. Propuso «explicar el carácter no adaptativo de tal conducta en términos de su percepción de la situación y su expectativa de lo que es probable que suceda» (cursiva añadida, 195I). Esta hipótesis no solo excluye la emocionalidad y la ideología de la explicación de las conductas, sino que, lo que es más importante, se servía de la anticipación del futuro para explicar el cambio de conducta en el presente.

Basado en esta hipótesis, el experimento del pensamiento fue diseñado para permitir un orden concreto de organización del espacio-tiempo para que surgiera con antelación un problema y su solución.

Se llevaron a cabo los experimentos con grupos de personas, de I5 a 2I sujetos en cada grupo. Los sujetos tenían que sacar conos de una botella de vidrio; se entregó a cada sujeto un trozo de cuerda a la que se ató un cono. Se precisaba la colaboración de los sujetos para que pudieran salir los conos; la disposición física facilitaba la aparición de «atascos» en el cuello de la botella. Solo podía salir un cono cada vez; incluso cuando coincidían dos conos en el cuello de la botella esto impedía que pudieran salir ambos.

Los «atascos» se transfirieron metafóricamente al cuello de la botella (Fig. III) mediante la materialización del experimento del pensamiento. Los materiales tuvieron que ser ajustados en varias ocasiones hasta que finalmente hicieron lo que el experimento de pensamiento requería. Por ejemplo, el cono tenía que ser de aluminio y no de plástico (debido a la escasez de la posguerra) o de madera (demasiado fácil de atascar), reduciendo el tamaño de la boca de salida y protegiendo el vidrio con cinta de goma, utilizando una almohadilla de goma esponjosa para

6 Este experimento ha sido adaptado incluso transnacionalmente en $3^{\text {rd }}$ grade Chinese/Ideological textbook (national version) in China at least since the mid 1990 s. 
proteger el fondo de la botella, introduciendo un tubo de goma para conducir el agua y dando cera al sedal para evitar que se enredaran. Por lo tanto, la situación y el problema quedaron representados de una forma específica que no solo estaba condicionada por la hipótesis de los investigadores, sino también por las cualidades físicas y económicas de los materiales experimentales para hacer de la solución del problema una forma muy particular de actuar.

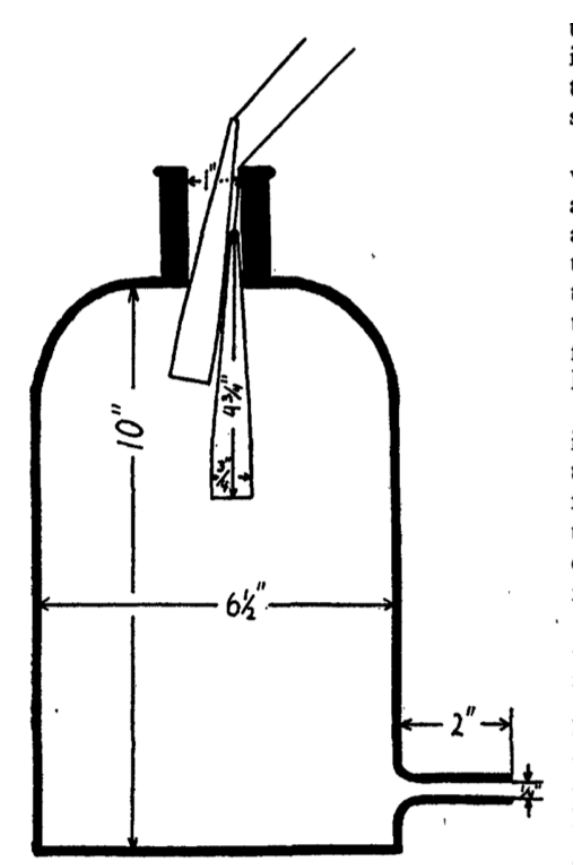

FIG. III. Botella experimental de Mintz. «Comportamiento humano no adaptativo» (I95I).

Para conseguir que los individuos tengan diferentes percepciones del futuro, Mintz utilizó como variable una estructura de recompensa basada en el tiempo. Se aceptaron los premios como incentivos que podían convertir el juego de la botella en una competición individual o en una cooperación para competir en grupo. Basándose en resultados experimentales, Mintz concluye su teoría de que la cooperación para la competición en grupo lograría una intervención más ordenada y, por lo tanto, resolvería el problema, mientras la competición individual conduciría al pánico y al desorden o, todavía más serio, al desastre. Sin embargo, como se ha demostrado antes, la equivalencia entre cooperación y orden para resolver el problema -sacar un cono a la vez-formaba parte de la constitución conceptual-material de los aparatos del experimento. En la conclusión del experimento, la equivalencia en el experimento de pensamiento fue sustituida por una conexión causal entre la anticipación del futuro y la resolución del problema que se consideró empíricamente probado por los participantes individuales. 


\subsection{Movimiento de la nave espacial Tierra}

Sin embargo, el fantasmagrama de un miembro de la multitud en un tiempo espacial finito, junto a su efecto de bucle, no solo entra y sale de los laboratorios de los psicólogos sociales. A partir de mediados de los años 1960, se transformó en un imaginario planetario y, con el desarrollo de la tecnología aeroespacial, se inventó la imagen de una Tierra global aislada, finita, íntima e interdependiente. Académicos de diferentes disciplinas, como economía, historia del mundo, geografía, ciencia aeroespacial, relaciones internacionales, se unieron al movimiento de la nave espacial Tierra (ver, por ejemplo, Ward, I966; Boulding, I971; Fuller, 1969; NASA, 1968; Asimov, 1974; AFSC, 1975).

En Spaceship Earth de Barbara Ward (1966), primera monografía escrita sobre este imaginario, y ampliamente difundida entre diferentes disciplinas, se definió la «comunidad humana» por su potencial destructivo de todos a la vez: «Si todos nosotros podemos ser destruidos, juntos, en dos o tres acciones de incineración grandilocuente, entonces somos vecinos». La intimidad y la interdependencia del planeta Tierra fueron concebidas como situaciones que producen conflictos/desastres, como «si alguien se enfadara o emborrachara en un submarino y buscara los controles del mismo. Si algún miembro de la raza humana se emborracha a bordo de nuestra nave espacial, todos tenemos problemas». Estas metáforas que se utilizaron para imaginar una crisis planetaria de los humanos no pretendían tanto buscar el mundo real como inducir al miedo y la ansiedad para forzar inmediatamente la intervención del mundo real.

Varias adaptaciones del imaginario de Ward fueron transferidas a los juegos de simulación y al currículum escolar sobre la nave espacial Tierra en la formación de expertos en los estudios sociales, educación internacional y ciencia de la educación de los Estados Unidos en los años 1970 (ver, por ejemplo, Nesbitt, 1968; King, 1969; Hawkhill, 1973; Bybee, 1979). Uno de estos programas experimentales se aplicó en la Escuela del Laboratorio Elemental del Colorado State College en 1969. A continuación, se describe cómo los niños simularon su supervivencia en una nave espacial, de la que podemos ver que los fantasmagramas anteriores vuelven a aparecer.

Los niños eran los pasajeros y la tripulación, a bordo de una nave espacial. Utilizando una transparencia que proyectaba un dibujo del interior de la nave, y una cinta de audio, se construyó el entorno. A medida que el grupo viajaba por el espacio la cinta de audio dramatizaba una secuencia de hechos que limitaban el suministro del aire, de la comida y del agua y creaban problemas por las condiciones de hacinamiento, circunstancias todas comprensibles con las que los jóvenes podían identificarse... Hablaron sobre las complicaciones que podrían surgir en un entorno limitado, de la necesidad de realizar esfuerzos cooperativos entre personas muy cercanas a cada uno, o dependientes unas de otras, sobre los tipos de conflicto interno que surgen cuando las personas están sujetas a incomodidad, privación, peligro y otras formas de estrés. (la cursiva es nuestra; King, 1971) 
En otro juego de simulación sobre el hambre en la nave espacial Tierra que fue creado por el American Friends Service Comité (1975), dos tercios de los miembros estaban «sometidos a incomodidades, privaciones, peligros y otras formas de estrés» y viajaban en el equivalente de la edad espacial de tercera clase. Procedían de países subdesarrollados y moraban en los guetos de las naciones ricas representadas en la sección de primera clase. La gran mayoría de los dos tercios de los viajeros hambrientos eran de color y la mayor parte de los bien alimentados eran blancos. Mediante el fantasmagrama de la supervivencia en botella, la vulnerabilidad de la «tercera clase» fue tomada en cuenta para la vulnerabilidad del planeta Tierra.

Se decía que la esperanza de la supervivencia humana dependía del pensamiento anticipatorio, de las capacidades de cálculo y control sistemático del sistema mundial total. R. Buckminster Fuller, veterano de la marina, teórico de sistemas y pensador de la anticipación, se convirtió en capitán de los seres humanos y en I968 escribió un libro titulado Operating Manual for Spaceship Earth. En el libro convirtió al Gran Pirata en el antiguo administrador de la tierra, que se arriesgó de forma global y dominó el cambio aprendiendo todo, haciendo predicciones a largo plazo, descubriendo cosas que previamente no tenían «valor» como recursos y conectándolas entre sí desde diferentes áreas para que los humanos pudieran compartir la tierra de manera equitativa. Se veía a científicos e ingenieros (Malthus, Darwin, los termólogos, Marx, que aceptaban que había suficiente para todos) como los servidores que ayudaron al Gran Pirata a descubrir el planeta y a construir un sistema de control mundial. Fuller justificó la conquista violenta y el robo de esos «perdedores» por la inteligencia del Gran Pirata al utilizar estrategias anticipatorias integrales. Al mismo tiempo, Fuller sostenía que esos «perdedores» serían torpes e indefensos al menos hasta que adquirieran la misma convicción y clarividencia que sus conquistadores. Aunque esos viejos «héroes» se habían extinguido, Fuller creía haber encontrado su sucesor: «Una nueva iniciativa metafísica, sin compromisos físicos, de integridad imparcial, podría unir el mundo. Podría y es probable que sería conseguida por medio de la resolución de problemas completamente impersonales de las computadoras» (p. 36).

La teoría de sistemas y las computadoras que la concretan lograron por adelantado su rol de salvador del mundo -ser el operador de la nave espacial- menos por lo que habían hecho que por su incorporación impersonal a la conquista histórica de los maestros de la nave y más tarde de la nave espacial. Inmediatamente después de que naciones de Asia, África y América Latina lograsen su independencia, teóricos de sistemas tanto de la ciencia como de la ciencia social del otro lado del Atlántico se unieron para debatir la manera de diagnosticar y controlar las nuevas amenazas emergentes de un sistema mundial cerrado. El objetivo principal de este enfoque era construir diferentes modelos de sistemas mundiales con ciencia, tecnología y matemáticas para describir cambios, identificar agentes causales y predecir y controlar sus futuras conductas.

Las narraciones de la nave espacial Tierra y la crisis global, incluyendo la superpoblación (Fig. IV), se basan en el fantasmagrama del crecimiento exponencial. 
El resultado se expande en la botella de Pearl. Los conos atascados en el cuello de la botella de Mintz. Un futuro de cautiverio y destrucción visto y no visto en una botella. Estos fantasmagramas de crisis estaban afectivamente impulsados por la aspiración a salvar al hombre y a la totalidad del planeta, y a controlar a los no cooperantes, a los participantes no adaptables para lograr el orden racional. Al mismo tiempo, el miedo y la ansiedad que ellos provocaban los convertía en la solución indispensable.

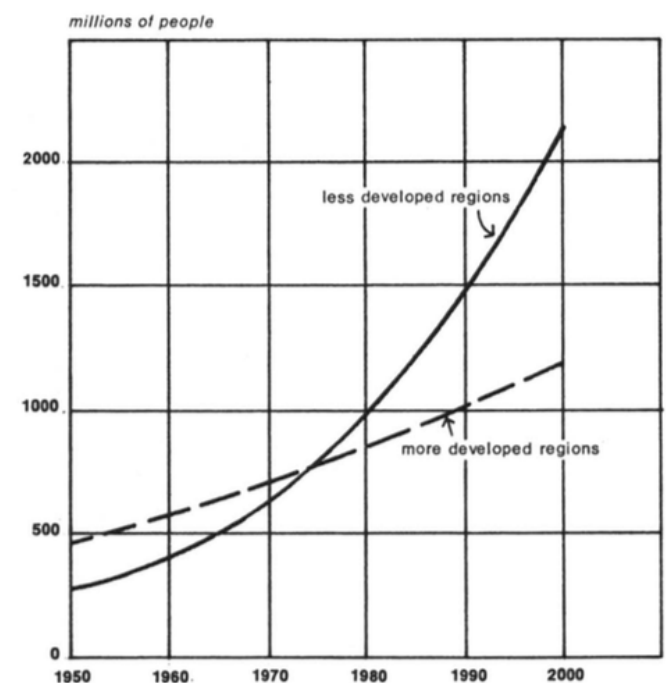

Total urban population is expected to increase exponentially in the less developed regions of the world, but almost linearly in the more developed regions. Present average doubling time for city populations in less developed regions is 15 years.

SOURCE: UN Department of Economic and Social Affairs, The World Population Situation in 1970 (New York: United Nations, 1971).

Fig. IV.

\section{Preparando la resolución de problemas para un milenio sin sorpresas} mediante la reforma del currículum de los Estados Unidos en la década de 1980

Los Institutos Internacionales de Análisis de Sistemas Aplicados (IIASA) y el Club de Roma, y otros muchos institutos internacionales de gobernanza y tecnología, patrocinaron el desarrollo de un modelo global computarizado para simular futuros escenarios de búsqueda de soluciones de precaución y de prevención a la posible perturbación de un estatus estable. El cambio del mundo que había sido anticipado mediante análisis de sistemas muy pronto ordenó un cambio de modelo de aprendizaje como medio para sobrevivir al cambio. Mientras el primer 
informe al Club de Roma, Limits to the Growth, realizó la primera y magnífica modelización global computarizada de la población en el laboratorio del MIT (Massachussetts Institute of Technology), el tercer informe al Club de Roma, No limits to learning (Botkin et al., I979), aplicó el pensamiento precautorio y anticipativo a la creación de la mente y la cultura humanas para sobrevivir en los años 1980. La preparación y la anticipación de la crisis futura para la supervivencia se convirtió así en un programa explícitamente cultural. Como plantearon los autores, «Colocar la supervivencia humana en primer plano como el primer objetivo del aprendizaje significa que no estamos debatiendo una cuestión metafísica; en su lugar, el aprendizaje se ha convertido en una cuestión de vida o muerte, y no sólo para la gente que se encuentra al límite de la subsistencia» (p. I4, la cursiva es mía).

Curiosamente, los autores de este libro cuestionaron directamente el enfoque cognitivo de la resolución de problemas que conceptualizaba al hombre como máquina y celebraba la autonomía distintiva del ser humano que se manifestaba por la anticipación y la participación. Introdujo el imaginario de la incertidumbre global y el choque inevitable como la nueva situación que requiere un aprendizaje anticipado en contraste con el «aprendizaje por choque», que se designó como una «fórmula para el desastre» (p. Io).

Se propusieron dos principios generales de aprendizaje anticipatorio. Uno era disponer de una colección exhaustiva de contextos y acontecimientos desconocidos para reducir la probabilidad de choque. El otro era también la «creación de nuevas alternativas donde antes no existían» para evitar las traumáticas y costosas lecciones del shock (p. 25). Guiado por estos dos principios, el aprendizaje anticipatorio pretendía un futuro estable ya no basado en una medida de probabilidad, sino en imaginar la posibilidad de situaciones desconocidas que se definían como amenazas y un conjunto de situaciones ya conocidas. Se definió a un aprendiz responsable como alguien que se atrevió a asumir riesgos, a controlar la crisis y a suscitar sorpresa en esas situaciones.

La anticipación de una actividad humana cada vez más devastadora y la idea de preparar a los alumnos y a las escuelas para crisis frecuentes se difundieron y desarrollaron aún más en los Estados Unidos en los años 1980. Shane y Tabler (I98I) invitaron a académicos de varias disciplinas de las ciencias naturales y sociales a debatir cómo elaborar un currículum para el nuevo milenio con la anticipación de «una era de agitación», que volvió a hacerse mediante informes futuristas estadounidenses, como por ejemplo The Global 2000 Report, through the 80's, the crucial years: 1986-2006, y otros similares.

El «salto evolutivo» que se experimentó en la botella de Pearl y que fue simulado por las computadoras de los científicos del MIT fue reformulado como «crisis de crisis» por uno de los panelistas, el biofísico Johan Platt, con un imaginario semejante al del pánico inducido por la multitud que se concretó en la botella de Mintz: «El alcalde de una gran ciudad suele poder afrontar emergencias como una fuerte nevada, huelgas de policías o de bomberos, o el descarrilamiento de un tren de cisternas llenas de productos químicos. Pero si el tren descarrila en medio 
de una nevada masiva en el momento en que policías y bomberos están en huelga, se produce de golpe una crisis total» (Shane y Tabler, 198I, p. 4I). Además, basado en los imaginarios de una nave espacial Tierra atestada de gente que crearon Ward y otros muchos, este debate también volvió a incidir en que la interdependencia de los recursos mundiales, las crecientes aspiraciones a mejorar las condiciones de vida y «tener más» $\mathrm{y}$ «tener menos» acceso al reparto conduciría a un aumento de los conflictos mundiales, ya que «en muchos casos no importa si la amenaza es real o supuesta; era percibir cómo la gente siente lo que lleva a los enfrentamientos, a los piquetes y, a veces, al frente de batalla» (p. 6, original en cursiva). En otras palabras, era el sentimiento de estar amenazado el que se hacía responsable de un futuro lleno de turbulencias.

En línea con este supuesto, se pidió a los jóvenes y adultos de Estados Unidos «tener el coraje de llegar a un acuerdo con la naturaleza y con su entorno psicológico, físico y sociológico, así como con sus grupos de iguales en el mundo», y se solicitó a los redactores del currículum que les ayudaran a «comprender conceptos de supervivencia, a entender cómo utilizarlos con prudencia, y a trabajar con otros para crear una sociedad mundial más humana y menos amenazadora» (p. 3I). Los educadores y responsables políticos actuales también podrían estar muy familiarizados con estos conceptos de supervivencia (véase Tabla I), pero con un título diferente: «conocimientos y habilidades para la innovación y la competitividad». Estos conceptos fueron creados para construir un futuro «sin sorpresas» y para «adaptarnos a lo inesperado o lo impredecible» (p. 46).

TABLA I. Conceptos de supervivencia (Shane y Tabler, 198r).

\begin{tabular}{|c|c|c|}
\hline & Ciencias Naturales & Ciencias Sociales \\
\hline $\begin{array}{l}\text { Conceptos de } \\
\text { supervivencia }\end{array}$ & $\begin{array}{l}\text { Límites, interdependencia, } \\
\text { ecocidio, entropía y conservación, } \\
\text { crecimiento explosivo de la pobla- } \\
\text { ción, método científico, evolución, } \\
\text { unidad de la naturaleza, ciclos de la } \\
\text { naturaleza, vulnerabilidad humana, } \\
\text { la necesidad de más investigación, } \\
\text { aprendizaje a lo largo de la vida, so- } \\
\text { brecarga informativa. }\end{array}$ & $\begin{array}{l}\text { Límites, interdependencia, } \\
\text { ecocidio, entropía y conservación, } \\
\text { crecimiento explosivo de la pobla- } \\
\text { ción, } \\
\text { método científico, evolución, uni- } \\
\text { dad de la naturaleza, ciclos de la } \\
\text { naturaleza, vulnerabilidad humana, } \\
\text { la necesidad de más investigación, } \\
\text { aprendizaje a lo largo de la vida, so- } \\
\text { brecarga informativa. }\end{array}$ \\
\hline
\end{tabular}

Para hacer superar a los estudiantes un futuro incierto, estos expertos invitados sugirieron estudiar y utilizar indicadores sociales para anticipar los cambios y después obtener un currículum de estas imágenes del futuro emergente para producir «un cambio evolutivo en la educación» (p. 77). Este paradigma de un currículum anticipatorio o un currículum derivado (Fig. V) fue desarrollado como una aplicación del cambio de paradigma de Kuhn (pp. 77-78). Sin embargo, la 
forma en que conceptualiza los cambios y los problemas fue exactamente lo que Kuhn (1962) entendía por «tradición», que se validó a sí misma transformando las contingencias en categorías existentes para la comprensión y el control. Por ejemplo, la planificación anticipada/derivada del currículum aceptó los cambios como tendencias lineales que podían reflejarse en los indicadores sociales y consideró la crisis como conflictos y la supervivencia como consenso entre lo viejo y lo nuevo, sin tener en cuenta si los criterios que los diferenciaban eran obsoletos. El supuesto epistemológico procedente del currículum convencional y del currículum innovador era el mismo. Esa era la brecha fundamental entre el pasado, el presente y el futuro

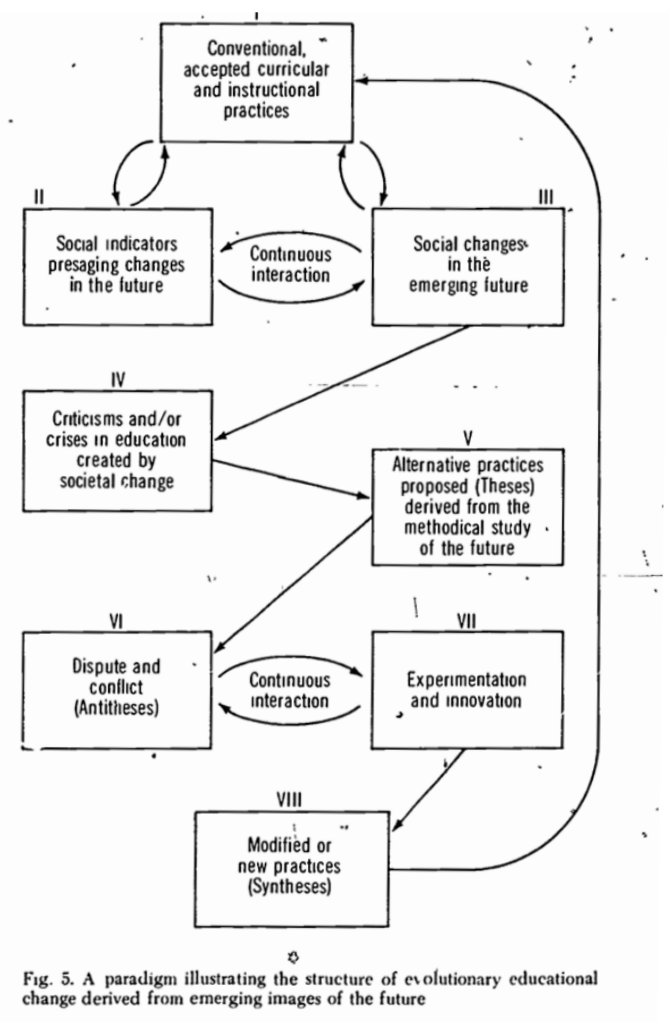

FIG. V. Planificación del currículum anticipatorio (Shane y Tabler, I98I, p. 78).

Sin embargo, el currículum anticipatorio/derivado aportó a los reformadores de la educación de los Estados Unidos -irónicamente la mayoría de miembros del comité de reforma no procedían del campo de la educación, sino de la ciencia, la tecnología, la ingeniería y los negocios- una razón para anticiparse a las crisis preparando a los estudiantes para las imágenes anticipadas del futuro. Provocada por el imaginario de una caída protencional de la nación, como clamaba $A$ 
Nation at Risk -Una nación en riesgo- Departamento de Educación de Estados Unidos, 1983), la heurística de la resolución de problemas se convirtió no solo en el contenido central de la New Math (ver, por ejemplo, NCTM, 1980; Romberg yTufte,1987; NSB, 1983; NCTM, 1989; Shoenfeld, 2007), sino también en los nuevos objetivos para la educación de los Estados Unidos en los años 1980 (Picus et al., 1983; Noble, 1989). Las preguntas sobre cómo conectar lo conocido y lo desconocido y cómo adaptarse a entornos inesperados se situaron en el corazón de la pedagogía de resolución de problemas.

De acuerdo con el principio del aprendizaje anticipatorio que he mencionado antes, aunque el objetivo era tratar con la incertidumbre, en los documentos de la reforma del currículum no se descartaban las situaciones rutinarias y los problemas comunes, sino que se consideraban esenciales para construir secuencialmente un repertorio de destrezas básicas y estrategias de resolución de problemas, sobre todo para los estudiantes de los primeros grados (NCTM, I980). Este repertorio de estrategias de aprendizaje en esencia significaba estrategias de control (Noble, 1989), ya que podía ser bien diseñado por instructores y utilizado por los alumnos en gran cantidad de situaciones familiares o en otras desconocidas.

Además de las estrategias cognitivas y visuales de «ver» el futuro, diferentes de la hipótesis de la conducta no adaptativa de Mintz, en el Nuevo Currículum de Matemáticas también se subrayaron los enfoques afectivos para la resolución de problemas (Romberg, 1987). An Agenda for Action-Una Agenda para la Acciónse hizo eco de la fanfarronada de Fuller sobre los Grandes Piratas al proponer «una mente abierta, una actitud de curiosidad y exploración, la complacencia en indagar» como el fundamento de la resolución de problemas (p. 3). La familiaridad con las situaciones y problemas también tenía importancia, en el sentido de proteger la confianza de los estudiantes y mantenerlos motivados en el proceso de aprendizaje (Picus et al., 1983).

No obstante, existió debate sobre si el enfoque de la resolución de problemas debía ser segmentado o integrador, si el desarrollo cognitivo era fijo o modificable. Por ejemplo, Romberg y Tufte (1987) sostenían que más abstracción y generalización aportaría más flexibilidad a la resolución de problemas. Sin embargo, parecía existir un consenso entre ambas partes sobre el uso de varias técnicas de representación visual de los problemas como algo decisivo para la resolución de problemas (ver NCTM, 1980; Picus et al., 1983; Romberg y Tufte, 1987). Estas técnicas incluían imágenes, diagramas esquemáticos, modelos gráficos, analogías, observación de fenómenos y simulación de escenarios reales o naturales. Los investigadores hicieron hincapié en la revisión conceptual de los problemas mediante formas visuales, asumiendo que los estudiantes no podían percibir los problemas y mucho menos convertirse en solucionadores de estos hasta que pudieran «ver» el problema con claridad. En otras palabras, solo los problemas que pueden ser visualizados son solubles y enseñables. Por tanto, la representación visual no solo definía lo que en realidad se apreciaba como problema, sino que, al mismo tiempo, como muestra la tendencia actual de los Big Data, se convertía en equivalente a las soluciones de los problemas que definía. 


\section{Conclusión}

Los fantasmagramas de la crisis -la supervivencia de la multitud en un espacio tiempo finito- que se crearon, reconfiguraron y circularon en la II Guerra Mundial y después de ella no solo pautan el primer desarrollo de la educación internacional, la educación ambiental, sino que también se reencarnan en el discurso pedagógico actual de resolución de problemas, que también es considerado como el corazón de la innovación, de la competencia global y la alfabetización STEM. Más aún, la repetición diferente de estos fantasmagramas normaliza performativamente la crisis y la supervivencia para justificar las reformas sociales y educativas que orientan la vida y el aprendizaje de los niños como guerreros que tienen que luchar contra lo desconocido, pero que al mismo tiempo atrapa su futuro dentro de la representación de un espacio finito e íntimo. Los diseñadores del currículum continuamente recopilan tipos particulares de «contextos» que se supone que generan problemas e incertidumbres -lo que se supone que es peligroso- para que los estudiantes aprendan a superar la vergüenza y el miedo y a utilizar lo que ellos «tienen» para resolver diferentes problemas. Además, los videojuegos populares para jóvenes y orientados a la educación, como Minecraft, crean un contexto sin memoria y convierten en normales las prácticas coloniales y neocoloniales de producción de «situaciones» para la autoridad científica. La resolución de problemas se ha convertido en una forma de imaginar ${ }^{7}$ lo que es un acto de imaginación que transforma el espacio tiempo indeterminado en un objeto del sistema de control conceptual-material y al mismo tiempo crea un sujeto que no solo se adapta, sino que también concede validez a ese sistema de control. Cuando la resolución de problemas se convierte en otro fantasmagrama de la crisis que lo hace ordinario, podría «frustrar el cambio y convertir el presente... en un conjunto de actualizaciones en las que corremos para permanecer quietos en el mismo sitio» (Chun, 2016, p. 3)

\section{Bibliografía}

American Friends Service Committee (AFSC): Hunger on Spaceship Earth: Simulation Game, New York, Author, 1975.

Asimov, Issac: Earth: Our Crowded Spaceship, New York: Fawcett Crest, 1974.

BAKER, Bernadette: «To show is to know? The conceptualization of evidence and discourses of vision in social science and education research», Curriculum Inquiry, (2017). DOI: I0.1080/03626784.2017.1283593

Bales, R. y Flanders, N.: «Planning an Observation Room and Group Laboratory», American Sociological Review, i9(6) (1954), pp. 771-78I.

El término está tomado del libro The Imagineers of War: The Untold Story of DARPA, the Pentagon Agency That Changed the World (Weinberger, 2017). También coincide «accidentalmente» con Imagineering the Future de Walter Disney. 

LEI ZHENG

Bales, Robert Freed y Strodtbeck, Fred L. «Phases in Group Problem-Solving», Journal of Abnormal and Social Psychology, 46 (195I), pp. 482-495.

BARAD, K.: «Posthumanist performativity: Toward an understanding of how matter comes to matter», Signs: Journal of Women in Culture and Society, 28(3) (2003), pp. 80I-83I.

BARAD, K.: Meeting the Universe Halfway, Duke University Press, 2007.

Bonneuil, Christophe: «Development as Experiment: Science and State Building in Late Colonial and Postcolonial Africa, 1930-1970", Osiris, 2nd ser., I5 (2000), pp. 258-28I.

Botkin, J. W.; Elmandjra, M. y Malitza, M.: No Limits to Learning: Bridging the Human Gap: The Report to the Club of Rome, Pergamon Press, 1979.

Boulding, Kenneth: «The Economics of the Coming Spaceship Earth», en Holdren, John P. y EhrLich. Paul R. (eds.): Global Ecology, Readings toward a Rational Strategy for Man, I97I, pp. I80-187.

Bybee, Robert: «Science Education Policies for an Ecological Society: Aims and Goals», Science Education, 63(2) (1979), pp. 245-255.

Chun, Wendy: Updating to Remain the Same: Habitual New Media, Cambridge, MA, The MIT Press, 2016.

Erickson, Paul; Klein, Judy L.; Daston, Lorraine; Lemov, Rebecca; STURM, Thomas y GorDIn, Michael D.: How Reason Almost Lost Its Mind: The Strange Career of Cold War Rationality, Chicago, University of Chicago Press, 2013.

Fuller, R. Buckminster: Operating Manual for Spaceship Earth, Carbondale, Southern Illinois University Press, 1969.

Hawkhill Associates: Spaceship earth, Madison, WI, Author, 2003.

KING, D. C.: International Education for Spaceship Earth, New York, Foreign Policy Association, 1971.

Kunn, T.: The Structure of Scientific Revolutions, Chicago, IL, University of Chicago Press, 1962.

Meadows, D. H.; Meadows, D. L.; Randers, J. y Behrens III, W. W.: The Limits to Growth: A Report for The Club of Rome's Project on The Predicament of Mankind, New York, Potomac Associates, 1972.

Mintz, A.: «Non-adaptive group behavior», The Journal of Abnormal and Social Psychology, 46(2) (195I), pp. I50-I59.

Murphy, M.: The Economization of Life, Durham, Duke University Press, 2017.

Nasa: Apollo 8 Mission Image - Earthrise, 1968. https://www.nasa.gov/image-feature/apollo-8-earthrise [3 de junio de 20I9].

National Academy of Engineering and National Research Council (NAE \& NRC): Engineering in $K$-I2 Education: Understanding the Status and Improving the Prospects, Washington, DC, The National Academies Press, 2009.

National Academy of Sciences, National Academy of Engineering, and Institute of Medicine (NAS, NAE \& IM): Rising Above the Gathering Storm: Energizing and Employing America for a Brighter Economic Future, Washington, DC, The National Academy Press, 2007.

National Council of Teachers of Mathematics: An Agenda for Action: Recommendations for School Mathematics of the 1980s, Reston, VA, NCTM, 1980.

National Council of Teachers of Mathematics (NCTM): Curriculum and Evaluation Standards for School Mathematics, Reston, VA, NCTM, 1989.

National Council of Teachers of Mathematics (NCTM): Principles and Standards for School Mathematics, Reston, VA, NCTM, 2000.

National SCIENCE BoARD: Educating Americans for the 2Ist century: a plan of action for improving mathematics, science, and technology education for all American elementary and secondary students so that their achievement is the best in the world by 1995: a report to the American people and the National Science Board, Washington, D.C., Author, I983. 
NesbitT, W.: «International Education for The Twenty-First Century [special issue]», Social Education, vol. XXXII, n. ${ }^{\circ} 7$ (1968), pp. 637-739.

Ngss Lead States: Next generation science standards: For states, by states, Washington, DC, The National Academy Press, 2or3.

Noble, D.: "Cocknit Cognition: Education, the Military and Cognitive Engineering", AI E Society, 3 (1989), pp. 27I-296.

Orr, Jackie: Panic Diaries: A Genealogy of Panic Disorder, Durham, NC, Duke University Press, 2006.

Picus, Larry et al.: Teaching Problem Solving: A Research Synthesis, Portland, Oreg, Northwest Regional Educational Lab. (ED 238 875), 1983.

Popkewitz, T.: «The Alchemy of the Mathematics Curriculum: Inscriptions and the Fabrication of the Child», American Educational Research Journal, 4I(I) (2004), pp. 3-34.

Romberg, T. y Tufte, F.: Chapter I5 «Mathematics Curriculum Engineering: Some Suggestions from Cognitive Science», en Romberg, Thomas A. y Stewart, Deborah M. (eds.): The Monitoring of School Mathematics: Background Papers. Volume I: The Monitoring Project and Mathematics Curriculum, Madison, WI, Wisconsin Center for Education Research, I987, pp. 7I-I08.

Schoenfeld, Alan: «Problem solving in the United States, 1970-2008: Research and theory, practice and politics», ZDM: The International Journal on Mathematics Education, 39(5) (2007), pp. 537-55I.

Schrader, A.: «Responding to Pfiesteria piscicida (the Fish Killer): Phantomatic Ontologies, Indeterminacy, and Responsibility in Toxic Microbiology», Social Studies of Science, 40(2) (2010), pp. 275-306.

Shane, H. y TABleR, M.: Educating for a New Millennium: Views of $\mathrm{I}_{32}$ International Scholars, Bloomington, Phi Delta Kappa Educational Foundation, 198r.

SHILLING, R.: Creating an Engine for Breakthrough Innovation in STEM Education, 20I4. URL: https://sites.ed.gov/oii/2OI4/I2/creating-an-engine-for-breakthrough-innovation-instem-education/

Troupe, T. K. y TejIDo, J.: What's the matter with the three little pigs? The fairy-tale physics of matter, North Mankato, MN, Picture Window Books, a Capstone imprint, 20I9.

US Department of Education, The National Commission on Excellence in Education: $A$ Nation at Risk: The Imperative for Educational Reform, 1983. https://www2.ed.gov/pubs/ NatAtRisk/index.html [3 de junio de 20I9].

US Department of Education, Office of InNovation and Improvement: STEM 2026: $A$ Vision for Innovation in STEM Education, Washington, DC, Author, 2016.

WARD, B.: Spaceship Earth, New York, Columbia University Press, 1966.

(Traducción de Elena Patricia Hernández Rivero, Universidad de Salamanca) 